\title{
Serious Airway Compromise in Rare Presentation of Posterior Tongue Base Abscess
}

\author{
Mohamed El-Amin ${ }^{1 *}$, Nick Ward ${ }^{2}$, Mohamed Elnor ${ }^{3}$ and Ian Sheppard ${ }^{4}$ \\ ${ }^{1}$ Core surgical training, CT2, Northwest Deanery, UK \\ ${ }^{2}$ Foundation doctor, FY2, Northwest Deanery, Manchester, UK
}

${ }^{3}$ ST3, Intensive care registrar, Severn Deanery, UK

${ }^{4}$ Consultant ENT, Pennine acute hospitals NHS trust, Manchester, UK

*Corresponding author: Mohamed El-Amin, Core surgical training, CT2, Northwest Deanery, UK.
Received Date: October 02, 2020

Published Date: October 21, 2020

\begin{abstract}
This is a rare case report of 37 years old lady presented with acute airway compromise after presenting a few times to the emergency department with worsening sore throat and been treated for tonsillitis. Patient oral examination was difficult due to severe trismus but no signs of tonsillitis. Naso-endoscopic examination showed severe swelling of the base of the tongue without any significant signs of recent trauma or other signs of the acute inflammatory process. Imaging showed large posterior tongue base abscess. Drainage of the abscess was achieved through the external approach. The patient recovered well and was discharged home on oral antibiotics. The condition was related to patent foramen caecum of small area which led to abscess formation due to canalisation of bacteria with no obvious source of trauma or oral infections.
\end{abstract}

Keywords: Tongue base; Abscess; Posterior tongue; Idiopathic; Foramen cecum

\section{Introduction}

Tongue abscesses especially posterior abscesses are very rare. It can be suggested that this is due to the tongue's resistance to infection. One of the reasons for this resistance to infection is that the tongue is constantly mobile, allowing the saliva to persistently cleanse the tongue [1]. Furthermore, the tongue is surrounded by a thick keratinized mucosa, preventing micro-organisms from penetrating into the parenchyma easily. On top of this, the muscle bulk of the tongue has a rich blood supply and lymphatic drainage. Although rare, it is important to understand and identify tongue base abscesses, as the resulting swelling may become life threatening due to upper airway compromise [2].

\section{Case Report}

A 37 years old lady presented to the GP clinic and A\&E multiple times and was treated for tonsillitis with oral penicillin following a 1-month history of worsening throat pain, tongue swelling, dysphagia and occasional breathlessness with a feeling of constriction in her airway. She was previously fit and well and a smoker of 3-4 cigarettes per day. However, her symptoms did not improve and on further presentation to A\&E was noted to have a 'hot potato' voice but no stridor or evidence of breathlessness. She could swallow her saliva but with difficulty. Her serum inflammatory markers were raised (Figure 1 ) and she was admitted to Rochdale Infirmary (22/04/17) for treatment with IV antibiotics and steroids, being referred to the ENT department 2 days later at Fairfield GH following no amelioration (Figure 1).

Upon ENT review, she had restricted neck movements to the right and trismus. There was swelling of the tongue on the right side, more localized to the posterior $1 / 3 \mathrm{rd}$. Tongue movements were restricted to the right and on protrusion deviation to the right was evident. On palpation the swelling was tender and firm. The floor of the mouth, buccal mucosa, tonsillar pillars and fossae were 
normal. No evidence of tonsillitis or quinsy was present. There was a diffuse anterior neck swelling involving levels I and II on the right side. Swelling was tender and soft to firm. Level II lymph nodes were palpable bilaterally. Flexible nasoendoscopy showed swelling of base of tongue on the right side with narrowed but adequate airway.

CT scan of neck showed a large heterogenous, multi-cystic collection $5 \mathrm{x} 4.9 \mathrm{x} 4 \mathrm{~cm}$ at the base of tongue on the right side, crossing the midline and involving the intrinsic tongue muscle. Peripheral enhancement and central areas of low density suggested an abscess, lymph node involvement was also noted bilaterally particularly in the right sided level II region. There was a mild indentation of the trachea at the level of the hyoid bone (Figure 2). She was listed for an incision and drainage via the neck under general anaesthesia. Awake fibre-optic intubation was attempted but failed so a tracheostomy was performed. Through an incision 2 fingers' breath below the right mandible, pus was drained and abscess was found to be deep and breaching the pharyngeal mucosa. The right submandibular gland was removed to gain access to the abscess. Culture of pus was reported as Streptococcus intermedius. Suitable antibiotics were administered according to the sensitivity (Figure 2).

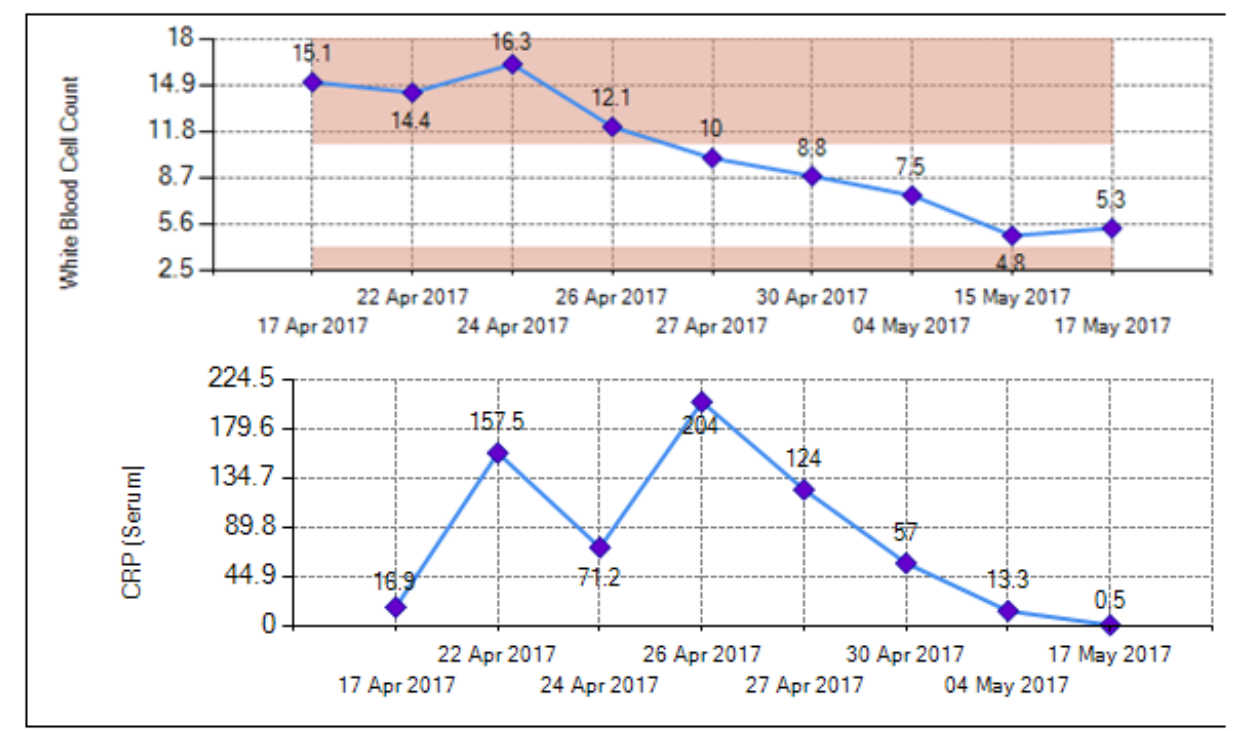

Figure 1: Graphs showing the trend in serum inflammatory markers, abscess incision and drainage was performed on 25/04/17.

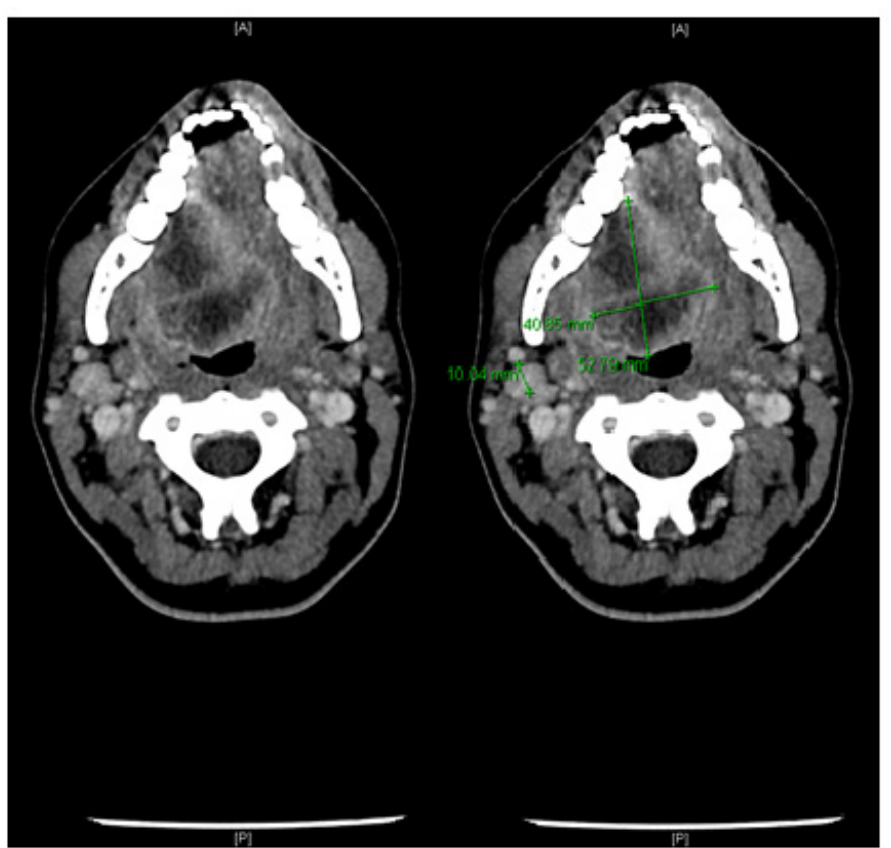

Figure 2: Axial CT images showing a heterogeneous multi-cystic collection at the base of the tongue with associated lymph node enlargement. 
Post-procedure and following a course of antibiotics, symptoms improved, and her tracheostomy was subsequently de-cannulated and she was discharged home, upon follow-up 2 weeks postprocedure, symptomatology on her tongue persisted and she still had right-sided hypoglossal nerve weakness. There was an indurated mass on the right ventral tongue extending down to the right tongue base, biopsy of which was performed and showed squamous cells and necrotic tissue which was confirmatory for abscess formation. No other abnormal cells were identified.

\section{Discussion}

When describing an abscess of the tongue, it is important to classify the areas into two categories: anterior and posterior [3]. The anterior two thirds of the tongue are known as the oral tongue, which is freely moving and is anterior to the circumvallate papillae. The tongue base is part of the oropharynx and is determined as the posterior third, located posterior to the circumvallate papillae. Infection of the base of the tongue may be caused by lingual tonsillitis, infected remnants of a thyroglossal cysts, or lower molar teeth infections. This is in contrast to anterior tongue abscesses, which are mainly cause by local trauma due to foreign bodies and jagged teeth [4]. That being said, a review of 12 cases of tongue abscesses by Sands et al found that in 10 out of the 12 cases, no specific cause was found. Despite the tongue's defences against infection that were mentioned earlier, tongue base abscesses may be predisposed by immunodeficiency states, chemotherapeutic drugs, and procedures that break the mucosa such as radiofrequency ablation $[1,5]$. Abscesses of the tongue base occur more frequently in men, which is thought to be due to a gender difference in smoking [4].

In the case reported, the patient was reviewed by a consultant Maxillo-facial surgeon who found no intra-oral mucosal abnormalities or dental aetiology that may have contributed towards abscess formation. The patient wasn't found to be immunologically compromised. Previous literature reporting on tongue base abscesses showed that sites are colonised by a mixed picture of micro-organisms [6]. Cultures have been shown to grow Streptococcus, Staphylococcus, anaerobes, as well as others. Other instances have been reported of colonisation with Actinomyces israelii, leading to actinomycosis, a chronic infection requiring long lasting treatment [7]. However, if the patient has been previously treated with antibiotics, no positive cultures may be seen [8].

\section{Conclusion}

Due to the location of tongue base abscesses, they may be challenging to diagnose [2]. The resulting infection and abscess formation leads to tongue protrusion, swelling, odynophagia, problems with speech and drooling, which develop over the course of hours to days. The resulting progressive dysphagia and dyspnoea mean that abscesses in this location should be managed as soon as possible. It is very uncommon presentation for the bas of the tongue abscess and it would require more research in the area with will help us to understand the pathophysiological process behind such condition.

\section{Acknowledgement}

None.

\section{Conflict of Interest}

None.

\section{References}

1. Ozturk M, Mavili E, Erdogan N, Cagli S, Guney, E (2006) Tongue abscesses: MR imaging findings. AJNR Am J Neuroradiol 27: 1300-1303.

2. Munoz A, Ballesteros AI, Castelo JB (1998) Primary lingual abscess presenting as acute swelling of the tongue obstructing the upper airway: diagnosis with MR. AJNR Am J Neuroradiol 19: 496-498.

3. Ozgur GT, Akdogan MV, Unler GK, Gokturk, HS (2015) A rare cause of acute Dysphagia: abscess of the base of the tongue. Case Rep Gastrointest Med 431738.

4. Sands M, Pepe J, Brown RB (1993) Tongue abscess: case report and review. Clinical infectious diseases 16: 133-135.

5. Tornari C, Won G, Arora A, Kotecha B (2015) A unique complication of radiofrequency therapy to the tongue base. International Journal of Surgery Case Reports 8: 9-12.

6. Carvalho TBOD, Fernandes AM, Sanches RA (2012) Tongue Abscess: Case Report. Arquivos Internacionais de Otorrinolaringologia 16: 126129.

7. Zamarro ML, Pérez ML, Soriano JM, Sanz GM (2005) Base tongue actinomycosis. Acta Otorrinolaringol Esp 56: 222-225.

8. Balatsouras DG, Eliopoulos PN, Kaberos AC (2004) Lingual abscess: diagnosis and treatment. Head Neck 26: 550-554. 